\title{
Three Reverse Adipofascial Cross Finger Flaps Used for Coverage of Raw Areas on Two Fingers of a Patient with Blast Injury of the Hand-Case Report
}

\author{
Medhat Emil*, Anood Alqaydi, Dalal AlMansoori, Dalia Medhat Habib, Marwa Alawadhi, \\ Abrar Alteneiji, Omar Alameri \\ Department of Plastic and Reconstructive Surgery, Zayed Military Hospital, Abu Dhabi, United Arab of Emirates \\ Email: *medhatemil1@hotmail.com
}

How to cite this paper: Emil, M., Alqaydi, A., AlMansoori, D., Habib, D.M., Alawadhi, M., Alteneiji, A. and Alameri, O. (2020) Three Reverse Adipofascial Cross Finger Flaps Used for Coverage of Raw Areas on Two Fingers of a Patient with Blast Injury of the Hand-Case Report. Modern Plastic Surgery, 10, 75-81.

https://doi.org/10.4236/mps.2020.103009

Received: May 23, 2020

Accepted: June 27, 2020

Published: June 30, 2020

Copyright $\odot 2020$ by author(s) and Scientific Research Publishing Inc. This work is licensed under the Creative Commons Attribution International License (CC BY 4.0).

http://creativecommons.org/licenses/by/4.0/

\begin{abstract}
We report a case of blast injury to the left hand which resulted in fractures of the fingers with exposure of bones and joints of the phalanges. We used three reverse adipofascial cross finger flaps raised at the same time from 2 fingers to reconstruct adjacent fingers of the patient. The patient recovered well postoperatively and had good range of movement of the fingers. This avoided the complications of the use of regional or distal flaps. To our knowledge, this is the first case reported in which three reverse adipofascial cross fingers flaps are raised at the same time, two of them from an injured finger, to cover three raw areas on two fingers of a patient.
\end{abstract}

\section{Keywords}

Adipofascial Flap, Reverse Cross Finger Flap, Blast Injury Hand, Simultaneous Flaps Fingers

\section{Introduction}

The first article that dealt with cross-finger flap was published in the year 1950 by Micheal Gurdin and John W. Pangman after the 2nd world war. They termed the procedure as "trans-digital flap". It was carved more on the lateral and volar surfaces of the donor digit [1]. Ten years later, Hoskins DH, published an article in which he described the design of the classic cross finger flap [2].

In 1978, Ivan Pakiam described the adipofascial cross finger flap [3] and further modifications took place in the design and shape of the flaps along the his- 
tory [4]. In this case report we used 3 reverse adipofascial cross finger flaps to reconstruct 2 fingers with exposed bones and joints in a patient of war blast injury.

\section{Case Report}

A 27 years old young soldier was involved in a mine blast injury during the Yemen war which resulted in crush injury of his left hand, burns with multiple foreign bodies of the left upper limb and intraoccular foreign bodies. The patient was initially stabilized in the field hospital and transferred next day to the national military hospital for further management.

On examination, the patient was found having facial burns with foreign bodies in both the globes. He sustained also burns of the left shoulder and arm with multiple puncture wounds and lacerations. He had bad crush injury of the left hand with amputated terminal phalanx of the left thumb and the amputated stump had already been closed with sutures in the field hospital. The patient had also multiple fractures of the left hand with fracture proximal, middle and terminal phalanges of the left index finger and intraarticular fracture of the proximal interphalangeal joint. There was loss of the central slip of the extensor tendon with exposure of the bones of the proximal and middle phalanges of the index finger at the dorsal aspect. The middle finger also had tissue loss at the dorsal aspect of the proximal interphalangeal joint with intraarticular undisplaced fracture head of the proximal phalanx. There was also injury of the nail beds of the left index, middle and little fingers (Figure 1 \& Figure 2).

\section{Operative Procedure}

The patient was taken on the same day of admission to the operation theater and removal of foreign bodies from the eyes was done by the ophthalmologists. Debridement of the dirty wounds and burnt area of the upper limb and hand was done by the Plastic Surgery team. He was taken again to the operation theatre two days later for further debridement and repair lacerations of the nailbeds and arm wounds.

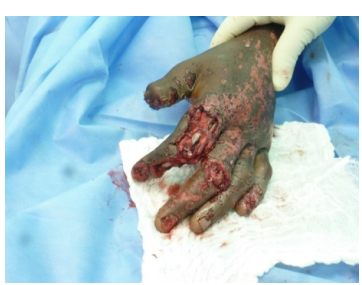

(a)

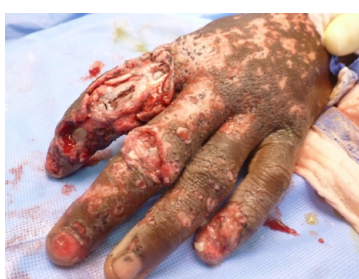

(b)

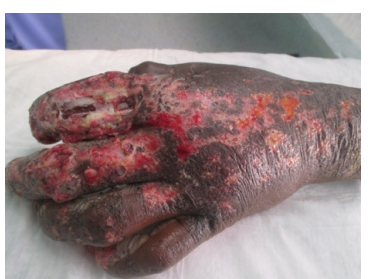

(c)

Figure 1. The hand injury sustained. (a) shows crush injury of the left hand with amputated terminal phalanx of the left thumb; (b) and (c) show exposure and fracture of the bones of the proximal and middle phalanges of the index finger with loss of the central slip of the extensor tendon at the dorsal aspect, tissue loss at the dorsal aspect of the proximal interphalangeal joint of the middle finger and injury of the nail beds of the index, middle and little fingers. 


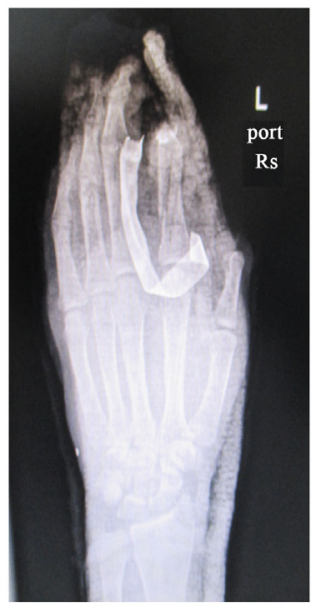

(a)

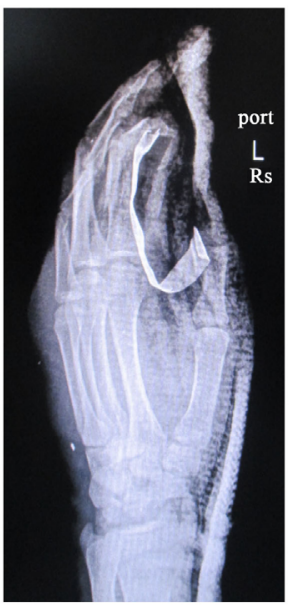

(b)

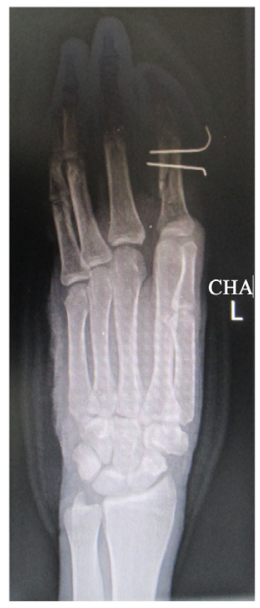

(c)

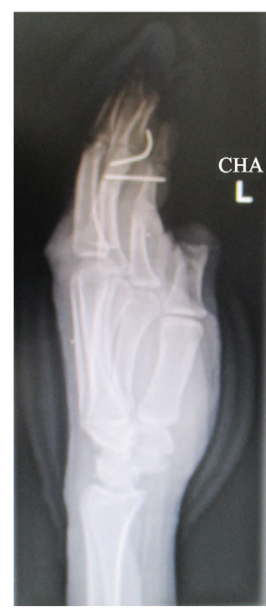

(d)

Figure 2. X-rays of the patient. (a) \& (b) Preoperative X-rays anteroposterior and oblique views; (c) \& (d) Postoperative X-rays anteroposterior and oblique views.

After two more days when the wounds were clean and the burn on the left upper limb was found improving conservatively, the patient underwent under general anaesthesia the coverage procedure after fixing the longitudinal fracture of the proximal phalanx of the index finger by two transverse K-wires.

Two reverse adipofascial cross finger flaps were designed from the dorsal aspect of the proximal and middle phalanges of the left middle finger to cover the dorsal aspects of the proximal and middle phalanges of the left index finger.

A third reverse adipofascial cross finger flap was designed from the dorsal aspect of the middle phalanx of the left ring finger to cover the dorsal aspect of the proximal interphalangeal joint of the left middle finger.

The technique used in each of the flaps was the same. Incisions were made longitudinally in the skin at the radial side of the donor finger and turned transversely proximally and distally at the dorsal aspect of each of the donor phalanges. Dissection was done between the dermis and the underlying tissues under magnification raising the skin as full thickness skin graft till the ulnar aspect of the finger. From this aspect, the underlying subcutaneous tissue was raised as a flap in the opposite direction and dissected from the underlying paratenon of the extensor tendon till the radial aspect of the finger placing the base of the flap next to the recipient finger. Following meticulous haemostasis, the flaps were reversed and sutured to the recipient sites with 5/0 Vicryl sutures. The donor defects were covered with the previously elevated full thickness skin. The reversed sides of the subcutaneous adipofascial flaps were surfaced with split thickness skin grafts harvested from the left thigh. Holes were made in the graft to prevent haematoma formation. A soft dressing was applied with volar Plaster of Paris slab (Figure 3).

The patient was put on dressings and 3 weeks later division of the three flaps was done. There was raw area at the ulnar aspect of the left index finger which was grafted. 

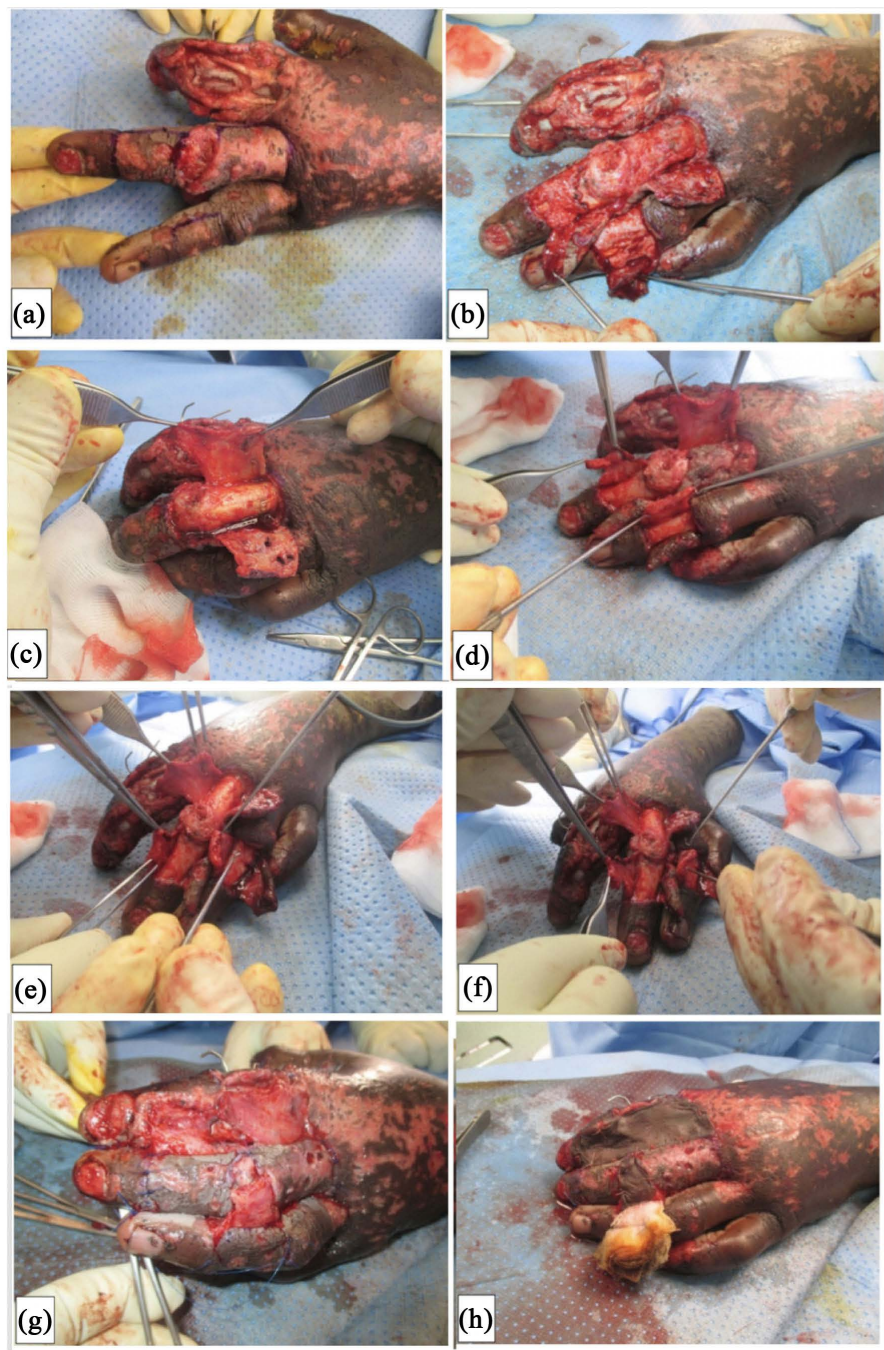

Figure 3. (a) shows K-wires fixation of the longitudinal fracture of the proximal phalanx of the index finger. Skin marking done; (b) Skin incised at the radial aspect of the middle and ring fingers and reflected and kept attached at the ulnar aspect of both the fingers; (c) The first reverse adipofascial flap at the proximal phalanx of the middle finger elevated from the ulnar aspect of the phalanx and reflected to cover the proximal phalanx of the index finger. Note the punctures in the flap and skin from the blast injury effect; (d)-(f) show the three reverse adipofascial cross finger flaps which were designed from the dorsal aspect of the proximal and middle phalanges of the left middle finger and the dorsal aspect of the middle phalanx of the left ring finger to cover the dorsal aspects of the proximal and middle phalanges of the left index finger and the dorsal aspect of the proximal interphalangeal joint of the left middle finger; ( $g$ ) shows the three flaps after reversing and suturing them to the recipient sites. The previously reflected skin is sutured back proximally and distally to its original site but not at the radial side of the phalanges where the pedicles of the flaps are; (h) shows split thickness skin graft surfacing the reversed side of the adipofascial flaps.

The patient was put on physiotherapy till he got good range of movement of his left hand. Extension of the left index finger was limited to 20 degrees but he had full flexion with normal grip of the hand. The postoperative follow up period was 103 days and the patient went back to his home country (Figure 4). 


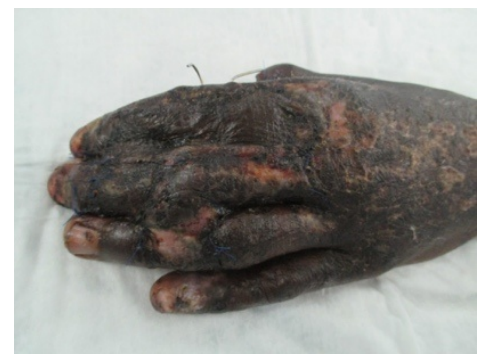

(a)

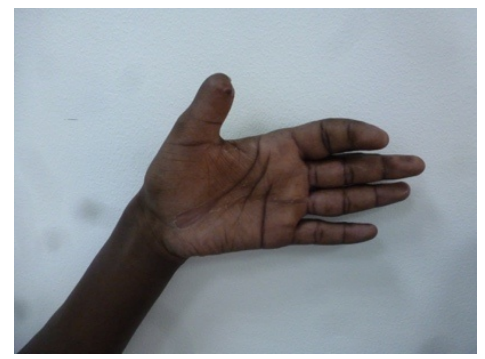

(c)

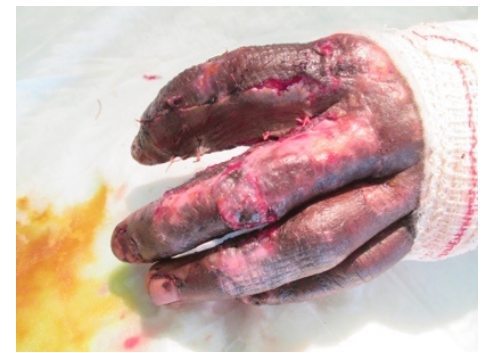

(b)

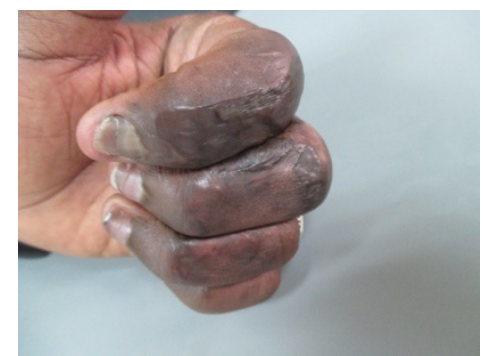

(d)

Figure 4. (a) Two weeks post operatively. The grafts are settling on the recipient's sites; (b) Three weeks post operatively; immediately post division of the three flaps and skin grafting of the ulnar aspect of the index finger; (c) \& (d) The left hand 3 months post operatively with slight limitation of extension with full flexion and normal grip of the hand.

\section{Discussion}

Many procedures are prescribed for coverage of raw areas of the hand. Distant flaps as the groin flap can be used but have the complications of stiffness of the shoulder and elbow joints [5]. Bulkiness of the flap is one of the drawbacks and subsequent defattening is usually required in most of the cases [6].

The reversed radial forearm flap can be used as fasciocutaneous or fascial flap with preservation of the radial artery but it has the disadvantage of having a large scar over the forearm and often the need for skin grafting to close the recipient site [7]. The reverse posterior interosseous flap is another option but in addition to the scarring on the forearm, it has the disadvantages of difficulty of dissection, inconstant anatomy of the artery, possibility of injury of posterior interosseous nerve and venous congestion of the flap. The flap also needs long time of elevation and a long learning curve [8].

Raising flaps from the hand itself can avoid such complications and provides tissues of similar texture and pliability. For these reasons we preferred to use the reverse adipofascial cross finger flaps rather than distant or regional flaps in our case.

The reverse adipofascial flaps are based on dorsal cutaneous branches from the proper digital arteries of the fingers. On his cadaveric study on 180 digits, Jefferson Silva showed that there are 2 constant branches in the proximal and middle phalanges from each proper digital artery and they have consistent sites of origin at predictable distances from the proximal interphalangeal joint for the long fingers [9]. We raised our three flaps; one from the proximal phalanx and 
two from the middle phalanges based on this anatomical concept. In spite of having many puncture wounds of the blast injury in our flaps, they all were viable and healed well which shows the good blood supply of the flaps.

The adipofascial flaps can be raised in different ways. They can be used as homodigital flaps within the same finger as turnover flaps or as heterodigital flaps from one finger to the adjacent one.

The homodigital flap can be used to cover the dorsal aspects of the middle [10] and terminal [11] phalanges of the fingers. The flap is elevated from the dorsal aspect of a phalanx and turned over to cover the phalanx distal to it. In our case, this type of flap could not be used as there was no soft tissue on the dorsal aspect of the proximal phalanx of the index finger to be turned over to cover the middle phalanx of that finger so we had to use a heterodigital type of flaps i.e. flaps from adjacent fingers.

Heterodigital flaps can be formed of either only of the adipofascial tissue between the dermis of the overlying skin and the underlying paratenon over the extensor tendon of the phalanges or can be formed of that tissue with the overlying skin after de-epithelializing it [12]. In the last option skin graft is applied on both the turned over flap and the paratenon of the donor site as one piece.

We feel that this technique can have high possibility of implantation dermoid cyst formation if no adequate de-epithelialization of the flap is done. Also applying skin graft on both the donor and recipient digits may be unnecessary if only skin graft can be applied on the recipient digit in the procedure we used. This will avoid disfigurement of the donor digit and maintain its normal hairy skin shape. For these reasons we preferred that our flaps to be formed only of the tissue between the dermis of the overlying skin and the underlying paratenon over the extensor tendon of the phalanges. After turning the flap 180 degrees like a page of a book and flap insetting is performed on the recipient site, the previously elevated overlying skin is turned back to cover the donor site without the need to do skin grafting of the donor digit.

\section{Conclusion}

The reverse adipofascial cross finger flap is a reliable procedure for covering raw areas of adjacent fingers. It provides soft tissue of proper thickness with good pliability and keeps the upper limb free of stiffness of distant flaps. To our knowledge, this is the first case reported in which three of these flaps were used to cover raw areas on two fingers of a patient at the same time.

\section{Conflicts of Interest}

The authors declare that they have no conflict of interest.

\section{Compliance with Ethical Standards}

All procedures performed in this study involving human participant were in accordance with the international ethical standards. Approval from the hospital 
Scientific and Research Committee was obtained.

\section{Informed Consent}

Additional informed consent was obtained from the participant for whom identifying information is included in this article.

\section{References}

[1] Gurdin, M. and Pangman, W.J. (1950) The Repair of Surface Defect of Fingers by Transdigital Flaps. Plastic and Reconstructive Surgery, 5, 368871.

[2] Hoskins, H.D. (1960) The Versatile Cross-Finger Pedicle Flap. JBJS Case Connector, 1, 261-277.

[3] Pakiam, A.I. (1978) The Reversed Dermis Flap. British Journal of Plastic Surgery, 31, 131-135. https://doi.org/10.1016/s0007-1226(78)90062-0

[4] Fang, F. and Chung, K.C. (2014) An Evolutionary Perspective on the History of Flap Reconstruction in the Upper Extremity. Hand Clinics, 30, 109-122.

https://doi.org/10.1016/j.hcl.2013.12.001

[5] Graf, P. and Biemer, E. (1992) Morbidity of the Groin Flap Transfer: Are We Getting Something for Nothing? JPRAS, 45, 86-88.

https://doi.org/10.1016/0007-1226(92)90162-q

[6] Arner, M. and Möller, K. (1994) Morbidity of the Pedicled Groin Flap a Retrospective Study of 44 Cases. Scandinavian Journal of Plastic and Reconstructive Surgery and Hand Surgery, 28, 143-146. https://doi.org/10.3109/02844319409071192

[7] Hansen, A.J., Duncan, S.F.M., Smith, A.A., Shin, A.Y., Moran, S.L. and Bishop, A.T. (2007) Reverse Radial Forearm Fascial Flap with Radial Artery Preservation. Hand, 2, 159-163. https://doi.org/10.1007/s11552-007-9041-7

[8] El-Sabbagh, A.H., Zeina, A.A.El-M., El-Hadidy, Al-M. and El-Din, A.B. (2011) Reversed Posterior Interosseous Flap: Safe and Easy Method for Hand Reconstruction. Journal of Hand and Microsurgery, 3, 66-72. https://doi.org/10.1007/s12593-011-0042-y

[9] Braga-Silva, J. (2005) Anatomic Basis of Dorsal Finger Skin Cover. Techniques in Hand and Upper Extremity Surgery, 9, 000-000. https://doi.org/10.1097/01.bth.0000173726.99670.f0

[10] Naji, S.H. (2001) Dorsal Adipofascial Turnover Flap for Dorsal Defects of Finger Injury. Medical Journal of Babylon, 8, 67-72.

[11] Ozdemir, R., Kilinç, H., Sensöz, O., Unlü, R.E. and Baran, C.N. (2001) Innervated Dorsal Adipofascial Turnover Flap for Fingertip Amputations. Annals of Plastic Surgery, 46, 9-14. https://doi.org/10.1097/00000637-200101000-00003

[12] Robbins, T.H. (1985) The Use of De-Epithelialised Cross-Finger Flaps for Dorsal Finger Defects. British Journal of Plastic Surgery, 38, 407-409.

https://doi.org/10.1016/0007-1226(85)90253-x 